\title{
Finite-time fluctuations for the totally asymmetric exclusion process
}

\author{
Sylvain Prolhad* \\ Laboratoire de Physique Théorique, Université de Toulouse, UPS, CNRS, France
}

\begin{abstract}
The one-dimensional totally asymmetric simple exclusion process (TASEP), a Markov process describing classical hard-core particles hopping in the same direction, is considered on a periodic lattice of $L$ sites. The relaxation to the non-equilibrium steady state, which occurs on the time scale $t \sim L^{3 / 2}$ for large $L$, is studied for the half-filled system with $N=L / 2$ particles. Using large $L$ asymptotics of Bethe ansatz formulas for the eigenstates, exact expressions depending explicitly on the rescaled time $t / L^{3 / 2}$ are obtained for the average and two-point function of the local density, and for the current fluctuations for simple (stationary, flat and step) initial conditions, relating previous results for the infinite system to stationary large deviations. The final formulas have a nice interpretation in terms of a functional integral with the action of a scalar field in a linear potential.
\end{abstract}

Non-equilibrium statistical mechanics aims to explain the emergence at large scales of simple evolution laws for a few macroscopic variables from the microscopic dynamics of many degrees of freedom. Universality appears at the mesoscopic scale, when the system is large but still finite, as only essential features of microscopic randomness subsist in the fluctuations of the macroscopic variables.

In specific classes of systems with local microscopic dynamics, fluctuations of macroscopic observables such as the position of the interface in growth models, the current of particles in driven lattice gases or the free energy for directed polymers in random media, are described by KPZ 1] universality [2 4]. The amplitude of KPZ fluctuations grows as $t^{\alpha / z}$ in the early time regime and saturates at $\ell^{\alpha}$ in a region of space of size $\ell$, with a crossover on the time scale $t \sim \ell^{z}$. The roughness and dynamical exponents, $\alpha=1 / 2$ and $z=3 / 2$ in one dimension, were measured experimentally in growing bacterial colonies [5] and burning fronts [6]. Scaling functions obtained during the last 15 years from several exactly solvable models were also observed in turbulent liquid crystal experiments [7, 8] and in numerical studies of various models [9 11].

The one-dimensional totally asymmetric simple exclusion process (TASEP) 12, 13] is an interacting particle system that has been used as a basis for models of cellular molecular motors 14], traffic flow 15], chains of quantum dots [16], and whose fluctuations belong to KPZ universality. TASEP describes the movement of hard-core particles, with the exclusion constraint that each site is either occupied by a single particle or empty. Particles move with a continuous-time Markovian dynamics, hopping from any site $i$ to the next site $i+1$ with rate 1 if the destination site is empty. We consider in this letter TASEP with $N$ particles on a periodic lattice of $L$ sites, on the crossover time scale $t \sim L^{z}$ which describes the evolution of KPZ fluctuations from those of the infinite system to the ones in the non-equilibrium steady state.

TASEP is a stochastic integrable model [17] with generator equal to the non-Hermitian Hamiltonian of a twisted XXZ spin chain. The formal analogy with quantum integrability provides powerful Bethe ansatz tools to analyse the model. Furthermore, TASEP always re-

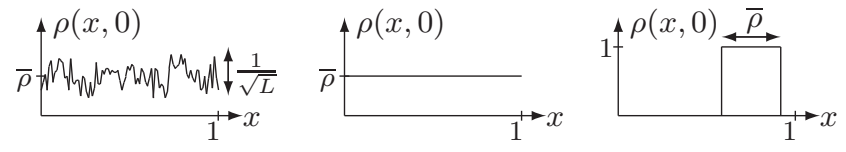

FIG. 1. Stationary, flat and step initial density profiles.

laxes to its unique stationary state, avoiding the complicated issues about thermalization occurring in quenches of quantum integrable systems with unitary evolution.

The probability $P_{t}(\mathcal{C})$ to observe at time $t$ a configuration $\mathcal{C}$ of TASEP obeys a master equation, and $P_{t}(\mathcal{C})=\left\langle\mathcal{C}\left|\mathrm{e}^{t M}\right| \mathcal{C}_{0}\right\rangle$ with $M$ the Markov matrix, for a system starting in configuration $\mathcal{C}_{0}$ at time 0 . We focus on three types of analytically tractable initial conditions: the stationary state where all configurations are equally likely, a flat configuration with equally spaced particles, and a step configuration with $N$ consecutive sites occupied. The average of the number of particles $\eta_{i}(\mathcal{C})$ (equal to 0 or 1 ) at site $i$ is given by

$$
\left\langle\eta_{i, t}\right\rangle=\sum_{\mathcal{C}} \mathbb{1}_{\left\{\eta_{i}(\mathcal{C})=1\right\}}\left\langle\mathcal{C}\left|\mathrm{e}^{t M}\right| \mathcal{C}_{0}\right\rangle,
$$

and similarly for the two-point function $\left\langle\eta_{0,0} \eta_{i, t}\right\rangle$.

On the hydrodynamic time scale $t \sim L$, the density profile $\rho(x, \tau)$, obtained at large $L$ from the occupation numbers $\eta_{i}$ with $x \simeq i / L$ and $\tau=t / L$, becomes deterministic at leading order: it is solution of Burgers' equation $\partial_{\tau} \rho+\partial_{x}(\rho(1-\rho))=0$. With $\bar{\rho}$ the conserved average density, the initial condition $\rho(x, 0)$ is equal in the stationary case to $\bar{\rho}$ plus a Brownian bridge of order $1 / \sqrt{L}$, in the flat case to $\bar{\rho}$, and in the step case to 1 in an interval of length $\bar{\rho}$ and 0 otherwise, see figure 1 .

In the first two cases, $\rho(x, \tau) \simeq \bar{\rho}$ at leading order for all $\tau$. In the step case, as with any generic initial condition, Burgers' equation generates shocks (discontinuities) after a finite time $\tau$. Only one shock remains at large $\tau$, moving with velocity $1-2 \bar{\rho}$. We consider in the following only the half-filled case $\bar{\rho}=1 / 2$ to avoid the need of a moving reference frame. Then $\rho(x, \tau) \simeq \frac{1}{2}-\frac{x-\kappa}{2 \tau}$ at large $\tau$ for $\kappa-1 / 2<x<\kappa+1 / 2$, with $\kappa \pm \frac{1}{2}$ the position of the shock. Our first main result (5), see also figure 3 a, 
describes the average density profile on the time scale $t \sim L^{3 / 2}$ when the sharp shock vanishes due to fluctuations in the microscopic model.

Multiple point correlations give informations about fluctuations beyond the hydrodynamics. They have been much studied for TASEP on the infinite line $\mathbb{Z}[18]$. In particular, the stationary two-point function of the density behaves [19] in the long time limit as $\left\langle\eta_{0,0} \eta_{i, t}\right\rangle-\frac{1}{4} \simeq$ $2^{-7 / 3} t^{-2 / 3} f_{\mathrm{KPZ}}\left(2^{-1 / 3} t^{-2 / 3} i\right)$. The Prähofer-Spohn scaling function $f_{\mathrm{KPZ}}$ [20] also describes the stationary twopoint function for propagating sound modes in generic one-dimensional Hamiltonian systems 21]. Our second main result (6), see also figure 4 is the two-point function for periodic TASEP on the time scale $t \sim L^{3 / 2}$. It agrees numerically with $f_{\mathrm{KPZ}}$ at short time.

KPZ universality also describes the current of particles of TASEP. The total (time-integrated) current $Q$ between time 0 and time $t$, equal to 0 when $t=0$, is incremented by 1 each time a particle hops anywhere in the system. The generating function of $Q$ verifies a deformed master equation 22]. With fugacity $\lambda$ conjugate to $Q$, one has $\left\langle\mathrm{e}^{\lambda Q / L}\right\rangle=\sum_{\mathcal{C}}\left\langle\mathcal{C}\left|\mathrm{e}^{t M(\lambda)}\right| \mathcal{C}_{0}\right\rangle$, where $M(\lambda)$ is obtained from multiplying by $\mathrm{e}^{\lambda / L}$ the non-diagonal entries of $M$ in configuration basis. The local (time-integrated) current $Q_{i}$, which counts only hops between site $i$ and $i+1$, is related to $Q$ by $Q / L=Q_{i}+\frac{1}{L} \sum_{j=1}^{N}\left(\left[x_{j}\right]_{i}-\left[x_{j}^{0}\right]_{i}\right)$. The $\left[x_{j}^{0}\right]_{i}$ 's and $\left[x_{j}\right]_{i}$ 's are integers between 1 and $L$ counting the positions of the particles from site $i+1$, respectively in the initial configuration $\mathcal{C}_{0}$ and in the final configuration $\mathcal{C}$. Calling $S_{i}$ the diagonal operator on configurations such that $S_{i}|\mathcal{C}\rangle=\sum_{j=1}^{N}\left(\left[x_{j}\right]_{i}-L / 2\right)|\mathcal{C}\rangle$, one has

$$
\left\langle\mathrm{e}^{\lambda Q_{i}}\right\rangle=\sum_{\mathcal{C}}\left\langle\mathcal{C}\left|\mathrm{e}^{-\frac{\lambda}{L} S_{i}} \mathrm{e}^{t M(\lambda)} \mathrm{e}^{\frac{\lambda}{L} S_{i}}\right| \mathcal{C}_{0}\right\rangle
$$

The statistics of $Q_{i}$ beyond its stationary value, $Q_{i} \simeq t / 4$ for the half-filled system, has been studied extensively for TASEP on the infinite line [18], and for a finite system in the long time limit 23]. Our third main result is the crossover (77)-(9) on the time scale $t \sim L^{3 / 2}$ between these two regimes, see also figures $3 \mathrm{~b}-3 \mathrm{H}$ and $5 \mathrm{~b}-5 \mathrm{H}$.

On the infinite line, current fluctuations are defined as $\xi_{t}^{\mathbb{Z}}=\left(Q_{0}-t / 4\right) / t^{1 / 3}$, with the amplitude of $\mathrm{KPZ}$ fluctuations growing as $t^{1 / 3}$. For stationary initial state, where sites are occupied independently with probability $1 / 2$, the statistics of $\xi_{t}^{\mathbb{Z}}$ is given 24] in the long time limit by $P\left(-\xi_{t}^{\mathbb{Z}}<u\right) \rightarrow F_{0}\left(2^{4 / 3} u\right)$ with $F_{0}$ the Baik-Rains distribution [25]. For flat initial condition, where every other site is occupied, the statistics is 26] $P\left(-\xi_{t}^{\mathbb{Z}}<u\right) \rightarrow F_{1}(4 u)$ with $F_{1}$ the Tracy-Widom distribution for the Gaussian orthogonal ensemble (GOE). Finally, for step initial condition, where only sites $i<0$ are occupied, one has [27] $P\left(-\xi_{t}^{\mathbb{Z}}<u\right) \rightarrow F_{2}\left(2^{4 / 3} u\right)$ with $F_{2}$ the Tracy-Widom distribution for the Gaussian unitary ensemble (GUE). Both $F_{1}$ and $F_{2}$ appeared initially in random matrix theory, where they describe statistics of the largest eigenvalue. The functions $F_{0}, F_{1}, F_{2}$ can be expressed in terms of the Hastings-Mcleod solution of Painlevé II equation. Alternative expressions in terms of Fredholm determinants with Airy kernels also exist. Numerical evaluations are available at [28], see figure 5.

For a large but finite system, on the other hand, KPZ fluctuations are independent of the initial condition in the long time limit, and their amplitude saturates at order $L^{\alpha}$. The current fluctuations of periodic TASEP are then defined as $\xi_{t}^{\text {st }}=\left(Q_{i}-t / 4\right) / \sqrt{L}$, which converges almost surely to $t /\left(4 L^{3 / 2}\right)$ for long $t$. Beyond this deterministic value, $P\left(\xi_{t}^{\mathrm{st}}=\frac{u t}{4 L^{3 / 2}}\right) \sim \exp \left(-t L^{-3 / 2} G_{\mathrm{st}}(u)\right)$ with $G_{\mathrm{st}}$ the Derrida-Lebowitz large deviation function [22], whose Legendre transform has an exact parametric expression. $G_{\text {st }}$ also describes the fluctuations of the free energy for a directed polymer in a random medium [29], of the height for an avalanche model [30], and of the current for open TASEP at the edge of the maximal current phase [31].

Our main results for density and current fluctuations at finite rescaled time $\tau=t /\left(2 L^{3 / 2}\right)$ follow from expanding (11), (2) over the eigenstates $r$ of $M(\lambda)$. Exact Bethe ansatz formulas 32, 33] allow to compute explicitly the large $L$ limit [34, 35], see supplemental material. The final expressions (5)-(9) have all roughly the form of discretized functional integrals with the action of a scalar field in a linear potential, and various operators inserted depending on the observable and the initial state:

$$
\sum_{r} \mathcal{A}_{r} \exp \left(\int_{-\infty}^{\nu_{r}} \mathrm{~d} v\left(\varphi_{r}^{\prime}(v)^{2}+\tau \varphi_{r}(v)\right)\right) .
$$

For current fluctuations, the upper limit $\nu_{r}$ of the integral in the action verifies $\varphi_{r}\left(\nu_{r}\right) \propto \lambda$, and $\varphi$ can thus be interpreted as a field conjugate to the current. The integral is regularized at $-\infty$.

When $\tau \rightarrow \infty$, only the stationary eigenstate $r=0$ contributes to (3). When $\tau \rightarrow 0$, the number of eigenstates $r$ contributing diverges, which makes it difficult to evaluate numerically our expressions (5)-(9) for very small $\tau$. With the values of $\tau$ accessible numerically, we are however able to confirm that the various observables connect as expected to the results for TASEP on $\mathbb{Z}$. A proof is missing at the moment. The Fredholm determinant (10), which is rather similar to known expressions for $F_{0}, F_{1}, F_{2}$, seems a good starting point.

From Bethe ansatz, each eigenstate of TASEP is fully characterized by $N$ momenta of fermionic quasi-particles, $k_{j}, j=1, \ldots, N$, integers or half-integers depending on the parity of $N$ and distinct modulo $L$. The stationary eigenstate corresponds to the Fermi sea $k_{j}=j-(N+1) / 2$ with Fermi momentum $k_{\mathrm{F}}=N / 2$, and each excited state $r>0$ contributing to the time scale $t \sim L^{3 / 2}$ is specified by two finite sets of half-integers $\mathbb{P}, \mathbb{H} \subset \mathbb{Z}+\frac{1}{2}$ describing momenta of particles and hole excitations, see figure 2 and supplemental material. Excitations on both sides of the Fermi sea are independent, thus both $\mathbb{P}$ and $-\mathbb{H}$ 


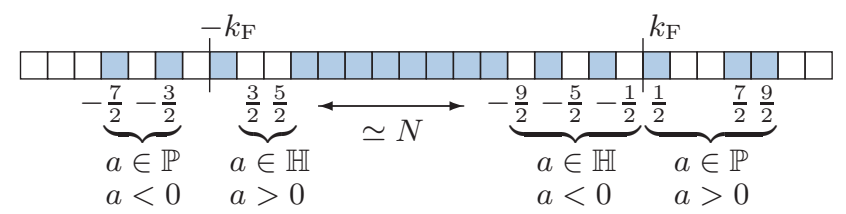

FIG. 2. Sets $\mathbb{P}$ and $\mathbb{H}$ for a choice of the $k_{j}$ (colored squares).

have $m_{r}=m_{+}+m_{-}$elements, with $m_{ \pm}$the number of elements with sign \pm . The stationary state corresponds to $\mathbb{P}=\mathbb{H}=\emptyset$. The total momentum of an eigenstate is $p_{r}=\sum_{a \in \mathbb{P}} a-\sum_{a \in \mathbb{H}} a$.

The field $\varphi_{r}$ is the derivative $\chi_{r}^{\prime}$ of $\chi_{r}$, defined by $\chi_{r}(v)=\chi_{0}(v)+\sum_{a \in \mathbb{P}} \omega_{a}^{3}(v) / 3+\sum_{a \in \mathbb{H}} \omega_{a}^{3}(v) / 3$ with $\chi_{0}(v)=-(2 \pi)^{-1 / 2} \mathrm{Li}_{5 / 2}\left(-\mathrm{e}^{v}\right)$ a polylogarithm and $\omega_{a}(v)=2(\operatorname{sgn}(a) \mathrm{i} \pi)^{1 / 2}\left(|a|+\operatorname{sgn}(a) \frac{\mathrm{i} v}{2 \pi}\right)^{1 / 2}$ the excitation with momentum $a$. The linear potential and kinetic part of the action come respectively from asymptotics of eigenvalues [34, 36] and eigenvectors 35] of $M(\lambda)$. They appear in (5)-(9) as $\tau \chi_{r}\left(\nu_{r}\right)$ and $D_{r}(\nu)^{2}$ with

$$
\begin{aligned}
D_{r}(\nu)= & \frac{\left(\frac{\mathrm{i} \pi}{2}\right)^{m_{r}^{2}}}{(2 \pi)^{m_{r}}}\left(\prod_{\substack{a, b \in \mathbb{P} \\
a>b}}(a-b)\right)\left(\prod_{\substack{a, b \in \mathbb{H} \\
a>b}}(a-b)\right) \\
& \times \exp \left(\lim _{\Lambda \rightarrow \infty}-m_{r}^{2} \log \Lambda+\int_{-\Lambda}^{\nu} \mathrm{d} v \frac{\chi_{r}^{\prime \prime}(v)^{2}}{2}\right),
\end{aligned}
$$

where $\log \Lambda$ cancels the divergence of the integral at $-\infty$.

We now state our main results. The density fluctuations $\sigma(x, \tau)=2 \sqrt{L}\left(\eta_{i}-\frac{1}{2}\right)$ at site $i=(\kappa+x) L$, for step initial condition $\mathcal{S}_{\kappa}$ with sites $\kappa L-N+1, \ldots, \kappa L$ occupied initially, are equal on average to

$$
\langle\sigma(x, \tau)\rangle_{\text {step }}=-2 \mathrm{i} \pi \sum_{r>0} \mathrm{e}^{2 \mathrm{i} \pi p_{r} x} \frac{p_{r} D_{r}^{2}\left(\nu_{r}\right) \mathrm{e}^{\tau \chi_{r}\left(\nu_{r}\right)}}{\chi_{r}^{\prime \prime}\left(\nu_{r}\right)},
$$

where $\nu_{r}$ is the solution of $\chi_{r}^{\prime}\left(\nu_{r}\right)=0$, and the summation is over all eigenstates except the stationary state. The function $\langle\sigma(x, \tau)\rangle_{\text {step }}$ is plotted in figure 3a along with results from simulations for a system of $L=1000$ sites averaged over $10^{7}$ independent realizations. The agreement is excellent: the $1 / L$ finite size corrections can hardly be seen. At short time, the density converges to the ramp centered at $x=0$ predicted from Burgers' equation. For stationary and flat initial condition, translation invariance implies $\langle\sigma(x, \tau)\rangle_{\text {flat } / \text { stat }}=0$.

Similarly, the stationary two-point correlation function $S(x, \tau)=\langle\sigma(0,0) \sigma(x, \tau)\rangle_{\text {stat }}$ for the density fluctuations at site 0 , time 0 and site $i=x L$, time $t$ is equal to

$$
S(x, \tau)=-(2 \pi)^{5 / 2} \sum_{r>0} \mathrm{e}^{2 \mathrm{i} \pi p_{r} x} \frac{p_{r}^{2} D_{r}^{2}\left(\nu_{r}\right) \mathrm{e}^{\tau \chi_{r}\left(\nu_{r}\right)}}{\mathrm{e}^{\nu_{r}} \chi_{r}^{\prime \prime}\left(\nu_{r}\right)},
$$

with $\nu_{r}$ again solution of $\chi_{r}^{\prime}\left(\nu_{r}\right)=0$. The two-point function is plotted in figure $4 \mathrm{a}$, along with results of simulations for a system of $L=100$ sites averaged over $10^{9}$ realizations. The agreement is good, except at very short
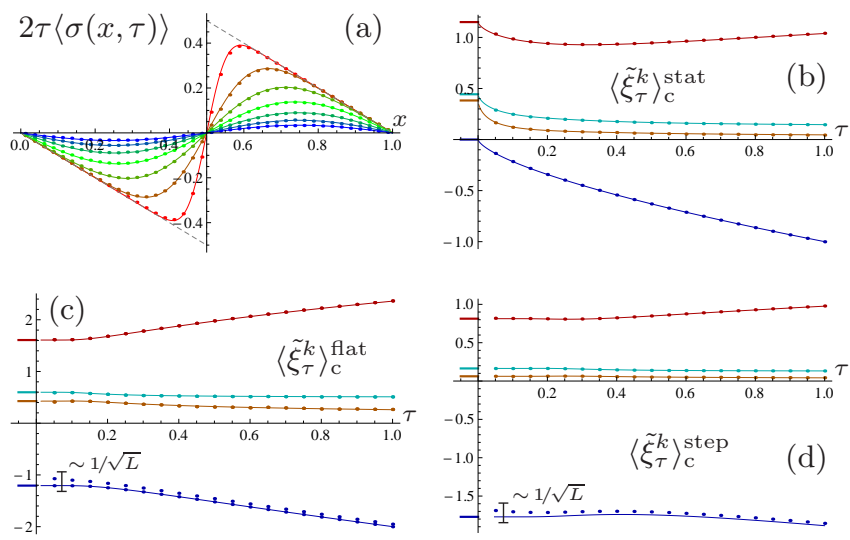

FIG. 3. (a) Average density profile for step initial condition at time $\tau=0.05$ (red curve, largest amplitude), $0.10, \ldots, 0.35$ (blue curve, smallest amplitude). The solid lines correspond to a numerical evaluation of the exact formula (5). The dots are the result of simulations. The dashed line is the ramp with a shock at $x=0.5$ obtained at small $\tau$ according to Burgers' equation. (b)-(d) Four first cumulants $\left\langle\tilde{\xi}_{\tau}^{k}\right\rangle_{\mathrm{c}}$ of the rescaled current fluctuations for stationary (b), flat (c) and step (d) initial condition with $x=0$, from numerical evaluations of (77)-(9). From top to bottom are plotted the variance (red), third (cyan) and fourth (orange) cumulants and the mean value (blue). The marks on the left represent the short time limits $F_{0}, F_{1}, F_{2}$. The dots are the result of simulations. The two sequences for $\left\langle\tilde{\xi}_{\tau}\right\rangle$ in (c) correspond to $Q_{i}$ at $i$ either initially occupied (lower dots) or empty (upper dots).
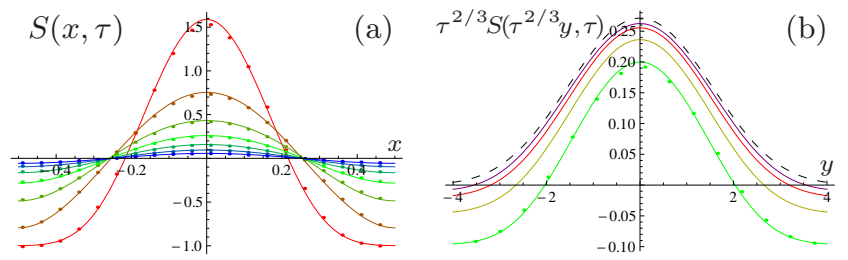

FIG. 4. (a) Stationary two-point function of the density $S(x, \tau)$ plotted as a function of $x$ for time $\tau=0.04$ (red curve, largest amplitude), $0.08, \ldots, 0.28$ (blue curve, smallest amplitude). The dots are the result of simulations. (b) Plots of $\tau^{2 / 3} S\left(\tau^{2 / 3} y, \tau\right)$ as a function of $y$ for smaller times $\tau=0.001$ (upper, solid purple curve), 0.003, 0.01, 0.03 (lower, solid green curve). The dots for $\tau=0.03$ are the result of simulations. The black, dashed curve is the Prähofer-Spohn scaling function $f_{\mathrm{KPZ}}(y / 2) / 2$, plotted from [28].

time when the scaling $t \sim L^{3 / 2}$ is not well verified for $L=100$. At short time $\tau$, our numerics seem to confirm the expected limit $\tau^{2 / 3} S\left(\tau^{2 / 3} y, \tau\right) \rightarrow f_{\mathrm{KPZ}}(y / 2) / 2$, see figure $4 \mathrm{~b}$. In contrast with the infinite line case, where the two-point function converges to 0 when the two points are far away, $S(x, \tau) \rightarrow-1$ when $x \neq 0$ and $\tau \rightarrow 0$ since the stationary measure of the periodic model is not exactly a product measure.

We define current fluctuations as $\xi_{\tau}=\frac{Q_{i}-t / 4-\mathcal{R} L}{\sqrt{L} / 2}$. The constant $\mathcal{R}$, which comes from the integration of Burgers' current between time 0 and infinity [37], is equal 
to 0 for stationary and flat initial condition, and the generating function of $\xi_{\tau}$ is given respectively by

$$
\left\langle\mathrm{e}^{s \xi_{\tau}}\right\rangle_{\text {stat }}=\sqrt{2 \pi} s^{2} \sum_{r} \frac{D_{r}^{2}\left(\nu_{r}\right) \mathrm{e}^{\tau \chi_{r}\left(\nu_{r}\right)}}{\mathrm{e}^{\nu_{r}} \chi_{r}^{\prime \prime}\left(\nu_{r}\right)}
$$

and

$$
\left\langle\mathrm{e}^{s \xi_{\tau}}\right\rangle_{\text {flat }}=s \sum_{r} \mathbb{1}_{\{\mathbb{P}=\mathbb{H}\}} \frac{\mathrm{i}^{m_{r}} D_{r}\left(\nu_{r}\right) \mathrm{e}^{\tau \chi_{r}\left(\nu_{r}\right)}}{\mathrm{e}^{\nu_{r} / 4}\left(1+\mathrm{e}^{-\nu_{r}}\right)^{1 / 4} \chi_{r}^{\prime \prime}\left(\nu_{r}\right)},
$$

with $\nu_{r}$ now solution of $\chi_{r}^{\prime}\left(\nu_{r}\right)=s$. For step initial condition $\mathcal{S}_{\kappa}$ with current counted at site $i=(\kappa+x) L$ modulo $L$ with $-1 / 2 \leq x \leq 1 / 2$, one has instead $\mathcal{R}=-|x| / 2$ and

$$
\left\langle\mathrm{e}^{s \xi_{\tau}}\right\rangle_{\text {step }}=s \sum_{r} \mathrm{e}^{2 \mathrm{i} \pi p_{r} x} \frac{D_{r}^{2}\left(\nu_{r}\right) \mathrm{e}^{\tau \chi_{r}\left(\nu_{r}\right)}}{\chi_{r}^{\prime \prime}\left(\nu_{r}\right)} .
$$

Comparing (5) and (9) gives the conservation law $\left\langle\partial_{\tau} \sigma\right\rangle+$ $\left\langle\partial_{x} j\right\rangle=0$ with instantaneous current $j=\partial_{\tau} \xi_{\tau}$.

The first cumulants of $\xi_{\tau}$, obtained from the generating functions (7)-(9), are plotted in figures $3 \mathrm{~b}-3 \mathrm{~d}$ along with results of simulations for a system of $L=1000$ sites averaged over $10^{7}$ realizations. The agreement is very good. We checked from simulations of smaller systems that the discrepancies for the mean value with step and flat initial condition are due to finite size corrections of order $1 / \sqrt{L}$ instead of $1 / L$ in all the other cases.

The stationary large deviations are reached at long time from the contribution $\mathrm{e}^{\tau \chi_{0}\left(\nu_{0}\right)}$ of the stationary state. The results on the infinite line are recovered numerically at short time: defining $\tilde{\xi}_{\tau}=-\xi_{\tau} / \tau^{1 / 3}$ for stationary initial condition, $\tilde{\xi}_{\tau}=-2^{2 / 3} \xi_{\tau} / \tau^{1 / 3}$ for flat initial condition and $\tilde{\xi}_{\tau}=-\left(\xi_{\tau}-\frac{x^{2}}{4 \tau}\right) / \tau^{1 / 3}$ for step initial condition, the cumulants of $\tilde{\xi}_{\tau}$ have a finite limit at small $\tau$. They converge respectively to the cumulants of $F_{0}, F_{1}$, and $F_{2}$ (with $x \neq 1 / 2$; numerics indicate a different distribution around the position of the shock $x=1 / 2$ in the step case).

The probability density $P_{\tau}$ of $\xi_{\tau}$, extracted from the generating function by Fourier transform $P_{\tau}(u)=$ $\int_{-\infty}^{\infty} \frac{\mathrm{d} s}{2 \pi} \mathrm{e}^{-\mathrm{i} s u}\left\langle\mathrm{e}^{\mathrm{i} s \xi_{\tau}}\right\rangle$, is plotted under rescaling by $t^{1 / 3}$ in figures $5 \mathrm{~b}-5 \mathrm{f}$. After some manipulations described in the supplemental material, the cumulative distribution function $F_{\tau}(u)=P\left(\xi_{\tau}>u\right)$ reduces in all cases to integrals of a Fredholm determinant,

$$
F_{\tau}(u)=\int \frac{\mathrm{d} \nu}{2 \mathrm{i} \pi} \alpha(\nu) \mathrm{e}^{\tau \chi_{0}(\nu)-u \chi_{0}^{\prime}(\nu)} \oint \frac{\mathrm{d} z}{2 \mathrm{i} \pi z} \operatorname{det}(\mathbb{1}-J) .
$$

The integral over $\nu$ goes from $\mathrm{e}^{-\mathrm{i} \theta} \infty$ to $\mathrm{e}^{\mathrm{i} \theta} \infty$ with $\pi / 5<\theta<\pi / 2$. The function $\alpha$ is respectively equal for stationary, flat and step initial condition to $\alpha_{\text {stat }}(\nu)=-\sqrt{2 \pi} \mathrm{e}^{-\nu} D_{0}^{2}(\nu) \partial_{u}, \quad \alpha_{\text {flat }}(\nu)=$ $D_{0}(\nu) \mathrm{e}^{-\nu / 4} /\left(1+\mathrm{e}^{-\nu}\right)^{1 / 4}$, and $\alpha_{\text {step }}(\nu)=D_{0}(\nu)^{2}$. The operator $J$ is defined from $K, L$ and $X$ acting on $\mathbb{Z}+1 / 2$, with kernels $K_{a, b}=\delta_{a, b} \exp \left(\frac{\tau \omega_{a}^{3}(\nu)}{3}+u \omega_{a}(\nu)\right)$,
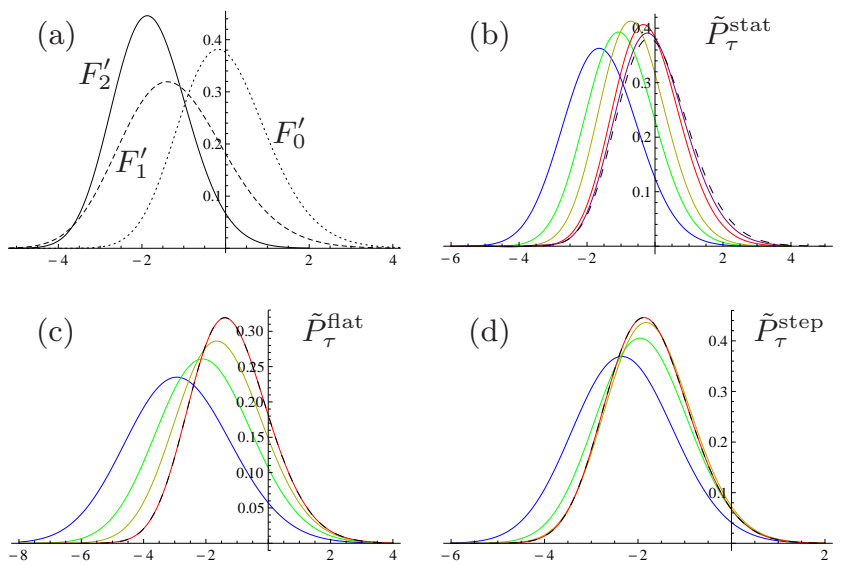

FIG. 5. (a) Probability density of Baik-Rains distribution $F_{0}$ (dotted line), GOE and GUE Tracy-Widom distributions $F_{1}$ (dashed line) and $F_{2}$ (solid line), evaluated from 28]. (b)-(d) Probability density $\tilde{P}_{\tau}$ of rescaled current fluctuations $\tilde{\xi}_{\tau}$ for stationary (b), flat (c), step initial condition at $x=0$ (d). The black, dashed curves are the short time limits $F_{0}, F_{1}, F_{2}$. The solid curves are finite time distributions at $\tau=2$ (lowest, blue curves), 1, 0.5, 0.1 (red), and 0.02 (purple) for (b).

$L_{a, b}=z^{\operatorname{sgn} a} f_{a}(\nu) f_{b}(\nu) /\left(\omega_{a}(\nu)+\omega_{b}(\nu)\right)$ with $f_{a}(\nu)=$ $\omega_{a}(\nu)^{-1 / 2} \exp \left(\int_{-\infty}^{\nu} \mathrm{d} v \frac{\chi_{0}^{\prime \prime}(v)}{\omega_{a}(v)}\right)$, and $X_{a, b}=\delta_{a, b} \mathrm{e}^{2 \mathrm{i} \pi a x}$. One has $J_{\text {stat }}=(K L)^{2}, J_{\text {flat }}=K^{2} L$ and $J_{\text {step }}=$ $X K L X^{-1} K L$. For an evolution conditioned on flat initial and final states, the Fredholm determinant is replaced by the infinite product $\prod_{a \in \mathbb{Z}+1 / 2}\left(1+z^{\operatorname{sgn} a} K_{a, a}^{2}\right)$ 37], and leads to large deviations at short time similar to the ones observed numerically in [9] for the total current.

Conclusions. The exact formulas derived in this letter for TASEP using the special structure of its Bethe ansatz generalize several known results for the infinite system and the non-equilibrium steady state of the periodic model. They provide the full crossover on the relaxation time scale $t \sim L^{3 / 2}$. Our main results (5)-(9) are expressed in a unified way and point to a field theoretic description of KPZ fluctuations in terms of a scalar field in a linear potential, $\varphi$, conjugate to the current.

Our results should hold for any model of onedimensional KPZ universality. It might be possible to recover them in other exactly solvable models, in particular stochastic Burgers' equation, using the replica method with asymptotics of eigenstates for the attractive $\delta$-Bose gas in finite volume, and ASEP, a generalization of TASEP where particles also hop backward with rate $q<1$, related to a twisted XXZ spin chain with anisotropy $\Delta=\left(q^{1 / 2}+q^{-1 / 2}\right) / 2>1$.

It would be interesting to generalize our results to the full statistics of time-dependent density and current profiles, and understand how the field $\varphi$ couples to an arbitrary initial condition $\sigma(x, 0)$ beyond the three specific initial states studied here. Another interesting but difficult question is the extension to the crossover between the 
far from equilibrium KPZ fluctuations and the EdwardsWilkinson fluctuations of an interface at equilibrium, corresponding for ASEP to the weakly asymmetric regime $q \rightarrow 1$ with $1-q \sim 1 / \sqrt{L}$.

sylvain.prolhac@irsamc.ups-tlse.fr

[1] M. Kardar, G. Parisi, and Y.-C. Zhang. Dynamic scaling of growing interfaces. Phys. Rev. Lett., 56:889-892, 1986.

[2] T. Kriecherbauer and J. Krug. A pedestrian's view on interacting particle systems, KPZ universality and random matrices. J. Phys. A: Math. Theor., 43:403001, 2010.

[3] J. Quastel and H. Spohn. The one-dimensional KPZ equation and its universality class. J. Stat. Phys., 160:965-984, 2015.

[4] T. Halpin-Healy and K.A. Takeuchi. A KPZ cocktailshaken, not stirred... J. Stat. Phys., 160:794-814, 2015.

[5] M. Matsushita, J. Wakita, H. Itoh, I. Ràfols, T. Matsuyama, H. Sakaguchi, and M. Mimura. Interface growth and pattern formation in bacterial colonies. Physica A, 249:517-524, 1998.

[6] M. Myllys, J. Maunuksela, M. Alava, T. Ala-Nissila, J. Merikoski, and J. Timonen. Kinetic roughening in slow combustion of paper. Phys. Rev. E, 64:036101, 2001.

[7] K.A. Takeuchi and M. Sano. Universal fluctuations of growing interfaces: Evidence in turbulent liquid crystals. Phys. Rev. Lett., 104:230601, 2010.

[8] K.A. Takeuchi, M. Sano, T. Sasamoto, and H. Spohn. Growing interfaces uncover universal fluctuations behind scale invariance. Sci. Rep., 1:34, 2011.

[9] D.S. Lee and D. Kim. Universal fluctuation of the average height in the early-time regime of one-dimensional KardarParisi-Zhang-type growth. J. Stat. Mech., 2006:P08014, 2006.

[10] K.A. Takeuchi. Statistics of circular interface fluctuations in an off-lattice Eden model. J. Stat. Mech., 2012:P05007, 2012.

[11] T. Halpin-Healy and Y. Lin. Universal aspects of curved, flat, and stationary-state Kardar-Parisi-Zhang statistics. Phys. Rev. E, 89:010103(R), 2014.

[12] B. Derrida. An exactly soluble non-equilibrium system: the asymmetric simple exclusion process. Phys. Rep., 301:65-83, 1998.

[13] O. Golinelli and K. Mallick. The asymmetric simple exclusion process: an integrable model for non-equilibrium statistical mechanics. J. Phys. A: Math. Gen., 39:12679$12705,2006$.

[14] T. Chou, K. Mallick, and R.K.P. Zia. Non-equilibrium statistical mechanics: from a paradigmatic model to biological transport. Rep. Prog. Phys., 74:116601, 2011.

[15] D. Chowdhury, L. Santen, and A. Schadschneider. Statistical physics of vehicular traffic and some related systems. Phys. Rep., 329:199-329, 2000.

[16] T Karzig and F. von Oppen. Signatures of critical full counting statistics in a quantum-dot chain. Phys. Rev. B, 81:045317, 2010.

[17] H. Spohn. Stochastic integrability and the KPZ equation. IAMP news bulletin, pages 5-9, April 2012.

[18] I. Corwin. The Kardar-Parisi-Zhang equation and universality class. Random Matrices: Theory and Applications, 1:1130001, 2011.
[19] M. Prähofer and H. Spohn. Current fluctuations for the totally asymmetric simple exclusion process. In V. Sidoravicius, editor, In and Out of Equilibrium: Probability with a Physics Flavor, volume 51 of Progress in Probability, pages 185-204. Boston: Birkhäuser, 2002.

[20] $f_{\mathrm{KPZ}}(y)$ is equal to $g^{\prime \prime}(y / 2) / 16$ in the notations of [19] and to $g^{\prime \prime}(y) / 4$ in that of [28].

[21] H. van Beijeren. Exact results for anomalous transport in one-dimensional Hamiltonian systems. Phys. Rev. Lett., 108:180601, 2012.

[22] B. Derrida and J.L. Lebowitz. Exact large deviation function in the asymmetric exclusion process. Phys. Rev. Lett., 80:209-213, 1998.

[23] B. Derrida. Non-equilibrium steady states: fluctuations and large deviations of the density and of the current. J. Stat. Mech., 2007:P07023, 2007.

[24] P.L. Ferrari and H. Spohn. Scaling limit for the spacetime covariance of the stationary totally asymmetric simple exclusion process. Commun. Math. Phys., 265:1-44, 2006.

[25] J. Baik and E.M. Rains. Limiting distributions for a polynuclear growth model with external sources. J. Stat. Phys., 100:523-541, 2000.

[26] T. Sasamoto. Spatial correlations of the 1D KPZ surface on a flat substrate. J. Phys. A: Math. Gen., 38:L549-L556, 2005.

[27] K. Johansson. Shape fluctuations and random matrices. Commun. Math. Phys., 209:437-476, 2000.

[28] M. Prähofer. http://www-m5.ma.tum.de/KPZ

[29] E. Brunet and B. Derrida. Probability distribution of the free energy of a directed polymer in a random medium. Phys. Rev. E, 61:6789-6801, 2000.

[30] A.M. Povolotsky, V.B. Priezzhev, and Chin-Kun Hu. The asymmetric avalanche process. J. Stat. Phys., 111:11491182, 2003.

[31] M. Gorissen, A. Lazarescu, K. Mallick, and C. Vanderzande. Exact current statistics of the asymmetric simple exclusion process with open boundaries. Phys. Rev. Lett., 109:170601, 2012.

[32] N.M. Bogoliubov. Determinantal representation of the time-dependent stationary correlation function for the totally asymmetric simple exclusion model. SIGMA, 5:052, 2009.

[33] K. Motegi, K. Sakai, and J. Sato. Long time asymptotics of the totally asymmetric simple exclusion process. J. Phys. A: Math. Theor., 45:465004, 2012.

[34] S. Prolhac. Spectrum of the totally asymmetric simple exclusion process on a periodic lattice - first excited states. J. Phys. A: Math. Theor., 47:375001, 2014.

[35] S. Prolhac. Asymptotics for the norm of Bethe eigenstates in the periodic totally asymmetric exclusion process. J. Stat. Phys., 160:926-964, 2015.

[36] L.-H. Gwa and H. Spohn. Six-vertex model, roughened surfaces, and an asymmetric spin Hamiltonian. Phys. Rev. Lett., 68:725-728, 1992.

[37] S. Prolhac. Current fluctuations and large deviations for periodic TASEP on the relaxation scale. J. Stat. Mech., 2015:P11028, 2015. 


\section{Supplemental material}

In this supplemental material, we briefly describe in the first section some aspects of the Bethe ansatz for TASEP, in particular asymptotics of eigenstates, some of them being new. In the second section, we derive the Fredholm determinant expression (10) for the cumulative distribution function of current fluctuations.

\section{LARGE $L$ ASYMPTOTICS OF TASEP EIGENSTATES FROM BETHE ANSATZ}

The Bethe ansatz gives each eigenvector of the deformed Markov matrix $M(\lambda)$ of TASEP with $N$ particles on a periodic lattice of $L$ sites as a linear combination of plane waves with $N$ complex momenta $q_{j}$. It reduces for TASEP to the determinant

$$
\langle\mathcal{C} \mid \phi\rangle \propto \operatorname{det}\left(\mathrm{e}^{\mathrm{i} q_{j} x_{\ell}} /\left(1-\mathrm{e}^{\mathrm{i} q_{j}-\lambda / L}\right)^{\ell}\right)_{j, \ell},
$$

with particles at positions $1 \leq x_{1}<\ldots<x_{N} \leq L$. Since particles are confined inside a box, the momenta take discrete values, solution of the Bethe equations

$$
\mathrm{e}^{\mathrm{i} q_{j}}\left(\frac{\left[1-\mathrm{e}^{\mathrm{i} q-\lambda / L}\right]}{1-\mathrm{e}^{\mathrm{i} q_{j}-\lambda / L}}\right)^{N / L}=\mathrm{e}^{2 \mathrm{i} \pi k_{j} / L}
$$

The set of numbers $k_{j}$, integers or half-integers depending on the parity of $N$ and distinct modulo $L$, fully characterizes the eigenstate. The geometric mean $\left[1-\mathrm{e}^{\mathrm{i} q-\lambda / L}\right]=\prod_{j=1}^{N}\left(1-\mathrm{e}^{\mathrm{i} q_{j}-\lambda / L}\right)^{1 / N}$ induces correlations between the momenta. The corresponding eigenvalue of $M(\lambda)$ is $E=\sum_{j=1}^{N}\left(\mathrm{e}^{-\mathrm{i} q_{j}+\lambda / L}-1\right)$, and the one of the translation operator is $\mathrm{e}^{2 \mathrm{i} \pi p / L}$ with total momentum $p=\sum_{j=1}^{N} k_{j}$.

Bethe ansatz naturally gives finite size expressions for the eigenstates. The main technical difficulty is usually to take the thermodynamic limit $L, N \rightarrow \infty$, which is needed in order to derive the main results (5)-(9). A first step was the exact calculation [S1] of the gap of the Markov matrix $M$, shown to scale as $L^{-3 / 2}$. A key observation is that lower eigenstates can be understood by a particle-hole picture, where the $k_{j}$ 's are interpreted as momenta of fermionic quasiparticles without spin. The stationary eigenstate corresponds to the Fermi sea $\left\{k_{j}^{0}=j-(N+1) / 2, j=1, \ldots, N\right\}$. Eigenstates close to it are obtained by exciting quasiparticles of momenta $k^{0},\left|k^{0}\right|<k_{\mathrm{F}}$ to $k,|k|>k_{\mathrm{F}}$ with Fermi momentum $k_{\mathrm{F}}=N / 2$. This creates vacancies in the Fermi sea, viewed as holes of momenta $-k^{0}$. It is conjectured [ $\left.\mathrm{S} 2\right]$ based on numerics for small systems that all the eigenstates of $M(\lambda)-\lambda / 4 \mathbb{1}$ with an eigenvalue of order $L^{-3 / 2}$ are generated by particle-hole excitations at a finite distance of the Fermi surface $\pm k_{\mathrm{F}}$, characterized by the two finite sets of half-integers $\mathbb{P}, \mathbb{H} \subset \mathbb{Z}+1 / 2$ of figure 2
The various known asymptotics of eigenvalues [S2] and eigenvectors $[\mathrm{S} 4, \mathrm{~S}, 5]$ of $M(\lambda)$ with finite rescaled fugacity

$$
s=\lambda \sqrt{L} / 2
$$

involve the elementary excitation $\omega_{a}(v)$ with momentum $a$, defined above (4), which verifies $\omega_{a}^{\prime}(v)=-1 / \omega_{a}(v)$ and is analytic for $v$ in the domain $\mathbb{D}=\mathbb{C} \backslash(\mathrm{i}[\pi, \infty) \cup$ $-\mathrm{i}[\pi, \infty)$ ) when $a \in \mathbb{Z}+1 / 2$. From these elementary excitations, one introduces above (4) the function $\chi_{r}$ with $\chi_{0}(v)$ given in terms of Hurwitz $\zeta$ function by $\chi_{0}(v)=$ $\frac{8 \pi^{3 / 2}}{3}\left(\sqrt{-\mathrm{i}} \zeta\left(-\frac{3}{2}, \frac{1}{2}+\frac{\mathrm{i} v}{2 \pi}\right)+\sqrt{\mathrm{i}} \zeta\left(-\frac{3}{2}, \frac{1}{2}-\frac{\mathrm{i} v}{2 \pi}\right)\right)$, analytic in $\mathbb{D}$. For $\operatorname{Re} v<0$ and for $\operatorname{Re} v>0,-\pi<\operatorname{Im} v<\pi$, $\chi_{0}(v)$ reduces to the polylogarithm given above (4). One also defines the solution $\nu_{r}$ of $\chi_{r}^{\prime}\left(\nu_{r}\right)=s$ for $\operatorname{Re} s \geq 0$, which induces a coupling between the quasiparticles. The quantity $\nu_{r}$ is singular for the stationary state when $s \rightarrow$ 0 , with $\nu_{0} \rightarrow-\infty$ and $\chi_{0}^{\prime \prime}\left(\nu_{0}\right) \simeq \mathrm{e}^{\nu_{0}} / \sqrt{2 \pi}$.

The Euler-Maclaurin formula allows to derive [S2] the large $L$ asymptotics $\left[1-\mathrm{e}^{\mathrm{i} q-\lambda / L}\right] \simeq \frac{1}{4}+\frac{\nu_{r}}{2 L}$ for the geometric mean of the Bethe roots, and the asymptotics

$$
E_{r}-\frac{\lambda}{4} \simeq \frac{\chi_{r}\left(\nu_{r}\right)}{2 L^{3 / 2}}
$$

for the eigenvalue. A key point for the derivation is that the Bethe roots $\mathrm{e}^{\mathrm{i} q_{j}}$ accumulate on a closed contour in $\mathbb{C}$, and Euler-Maclaurin's leading integral can be computed explicitly using residues.

The norm of the Bethe eigenstates of $M(\lambda)$, given in general by the Gaudin determinant, simplifies for TASEP [\$3]. The components of the eigenvectors for flat and step configuration reduce to Vandermonde determinants, and have been computed in the thermodynamic limit [S4, $\mathrm{S} 5]$ using the Euler-Maclaurin formula. We use the same normalization of the eigenvectors $\phi_{r}$ as in [S5]. It verifies $\left\langle\mathcal{C} \mid \phi_{r}\right\rangle=\left\langle\phi_{r} \mid \tilde{\mathcal{C}}\right\rangle$, where the configurations $\mathcal{C}$ and $\tilde{\mathcal{C}}$, corresponding respectively to particles at positions $1 \leq x_{1}<\ldots<x_{N} \leq L$ and $1 \leq \tilde{x}_{1}<\ldots<\tilde{x}_{N} \leq L$, are related by space reversal $\tilde{x}_{j}=L+1-x_{N+1-j}$. The scalar product is then equal for large $L$ to

$$
\frac{\Omega}{\left\langle\phi_{r} \mid \phi_{r}\right\rangle} \simeq \frac{\mathrm{e}^{\nu_{r}}}{\sqrt{2 \pi} \chi^{\prime \prime}\left(\nu_{r}\right)}
$$

where $\Omega=\left(\begin{array}{l}L \\ N\end{array}\right)$ is the total number of configurations. The component of the left eigenvectors for a flat configuration $\mathcal{F}$ is

$$
\left\langle\phi_{r} \mid \mathcal{F}\right\rangle \simeq \mathbb{1}_{\{\mathbb{P}=\mathbb{H}\}} \frac{\mathrm{i}^{m_{r}} \mathrm{e}^{-\nu_{r} / 4}}{\left(1+\mathrm{e}^{-\nu_{r}}\right)^{1 / 4}} .
$$

For a step configuration $\mathcal{S}_{\kappa}$ with sites $\kappa L-N+1, \ldots, \kappa L$ occupied initially, one has

$$
\left\langle\phi_{r} \mid \mathcal{S}_{\kappa}\right\rangle \simeq \mathrm{e}^{-\lambda L / 8} \mathrm{e}^{-2 \mathrm{i} \pi p_{r} \kappa} D_{r}\left(\nu_{r}\right),
$$

with $D_{r}$ defined in (4). All the expressions are understood as analytic in $\nu_{r} \in \mathbb{D}$. Note that there is a misprint 
in equation (35) of the published version of [\$5], with a global factor $\mathrm{i}_{r}^{2}$ missing.

The previous asymptotics are sufficient for studying an evolution conditioned on both initial and final configurations being flat or step [S5]. Relaxing the conditioning on the final state for the fluctuations of $Q_{i}$ requires the asymptotics of $\sum_{\mathcal{C}}\left\langle\mathcal{C}\left|\mathrm{e}^{-\frac{\lambda}{L} S_{i}}\right| \phi_{r}\right\rangle$. It can be obtained from the finite size Bethe ansatz formula (valid without assuming that the $q_{j}$ 's are solution of Bethe equations) $\sum_{\mathcal{C}}\left\langle\mathcal{C}\left|\mathrm{e}^{-\frac{\lambda}{L} S_{0}}\right| \phi_{r}\right\rangle \propto \operatorname{det} V[\mathrm{~S}[6]$ with $V_{j, k}=\sum_{\ell=0}^{j-1}(-1)^{\ell}\left(\begin{array}{c}L \\ \ell\end{array}\right) y_{k}^{\ell+1-j}$ for $1 \leq j<N, V_{N, k}=$ $-\sum_{\ell=N-1}^{L}(-1)^{\ell}\left(\begin{array}{c}L \\ \ell\end{array}\right) y_{k}^{\ell+1-N}$ and $y_{k}=1-\mathrm{e}^{\mathrm{i} q_{k}-\lambda / L}$. The operator $S_{0}$ precisely cancels a factor from the eigenvector expressed in terms of the $y_{k}$ 's, and makes the calculation of fluctuations possible for $Q_{i}$ but not for $Q$. The term $\ell=N-1$ can be removed from the sum for $V_{N, k}$ by linear combination with $V_{1, k}$. Rewriting $V_{N, k}=-y_{k}^{1-j}\left(1-y_{k}\right)^{L}+\sum_{\ell=0}^{N-1}(-1)^{\ell}\left(\begin{array}{c}L \\ \ell\end{array}\right) y_{k}^{\ell+1-N}$, expanding the row $j=N$ of the determinant, and doing further linear combinations to keep only the largest value of $\ell$ in each sum, $\operatorname{det} V$ reduces to a $N \times N$ Vandermonde determinant plus $N$ Vandermonde determinants of size $N-1$. Factorising everything leads to $\operatorname{det} V \propto\left(\prod_{j<k}\left(y_{j}-y_{k}\right)\right)\left(1-\sum_{j}\left(1-y_{j}\right)^{L} \prod_{k \neq j} \frac{y_{k}}{y_{k}-y_{j}}\right)$. Assuming now that the $y_{j}$ 's are solution of the Bethe equations, the second factor reduces to $1-\mathrm{e}^{-\lambda}$ after using the identity $\sum_{j=1}^{N} \prod_{k \neq j} \frac{y_{j}}{y_{j}-y_{k}}=1$. The asymptotics [S4] of the Vandermonde determinant in front leads to

$$
\frac{1}{\Omega} \sum_{\mathcal{C}}\left\langle\mathcal{C}\left|\mathrm{e}^{-\frac{\lambda}{L} S_{i}}\right| \phi_{r}\right\rangle \simeq \mathrm{e}^{2 \mathrm{i} \pi p_{r} i / L} \sqrt{2 \pi} s \mathrm{e}^{-\nu_{r}} D_{r}\left(\nu_{r}\right)
$$

Exactly the same asymptotics is found for $\left\langle\phi_{r}\left|\mathrm{e}^{\frac{\lambda}{L} S_{-i}}\right| P_{\text {st }}\right\rangle$ with $\left|P_{\text {st }}\right\rangle=\frac{1}{\Omega} \sum_{\mathcal{C}}|\mathcal{C}\rangle$ the stationary state.

Inserting the operator $\mathbb{1}_{\left\{\eta_{i}(\mathcal{C})=0\right\}}$ replaces $\operatorname{det} V$ by the determinant of another matrix $\tilde{V}$ [\$] 6 , defined by replacing $L$ by $L-1$ everywhere in the expression of $V$. One finds then $\operatorname{det} \tilde{V} \propto\left(\prod_{j<k}\left(y_{j}-\right.\right.$ $\left.\left.y_{k}\right)\right)\left(1-\sum_{j}\left(1-y_{j}\right)^{L-1} \prod_{k \neq j} \frac{y_{k}}{y_{k}-y_{j}}\right)$. Using the identity $\sum_{j=1}^{N} \frac{1}{1-y_{j}} \prod_{k \neq j} \frac{y_{j}}{y_{j}-y_{k}}=\prod_{j=1}^{N} \frac{1}{1-y_{j}}$, the Bethe equations imply that the second factor is equal to $1-$ $\mathrm{e}^{-L \gamma} \prod_{j=1}^{N} \frac{1}{1-y_{j}}$, and one has

$\frac{1}{\Omega} \sum_{\mathcal{C}} \mathbb{1}_{\left\{\eta_{i}(\mathcal{C})=0\right\}}\left\langle\mathcal{C} \mid \phi_{r}\right\rangle \simeq \frac{\sqrt{2 \pi}}{2 \sqrt{L}} \mathrm{e}^{2 \mathrm{i} \pi p_{r} i / L} 2 \mathrm{i} \pi p_{r} \mathrm{e}^{-\nu_{r}} D_{r}\left(\nu_{r}\right)$

at $\lambda=0$ for $r \neq 0$. In the stationary state, the right hand side is replaced by $1 / 2$. The asymptotics of $\frac{1}{\Omega} \sum_{\mathcal{C}} \mathbb{1}_{\left\{\eta_{-i}(\mathcal{C})=0\right\}}\left\langle\phi_{r} \mid \mathcal{C}\right\rangle$ is exactly the same.

Gathering all the large $L$ asymptotics above, one recovers the main results (5)-(9) of the letter.

\section{FREDHOLM DETERMINANT FOR THE CURRENT FLUCTUATIONS}

The Fredholm determinant (10) for the cumulative distribution function of current fluctuations is a consequence of an alternative expression for $D_{r}(\nu)$, obtained from (4) by computing explicitly the terms of the integral independent of $\chi_{0}$ using the identity $\partial_{v} \log \left(\omega_{a}(v)+\omega_{b}(v)\right)=$ $-\omega_{a}^{-1}(v) \omega_{b}^{-1}(v)$. It leads to the Cauchy determinant

$D_{r}(\nu)=\exp \left(\int_{-\infty}^{\nu} \mathrm{d} v \frac{\chi_{0}^{\prime \prime}(v)^{2}}{2}\right) \operatorname{det}\left(\frac{\mathrm{i} f_{a}(\nu) f_{b}(\nu)}{\omega_{a}(\nu)+\omega_{b}(\nu)}\right)_{\substack{a \in \mathbb{P} \\ b \in \mathbb{H}}}$

with $f_{a}(\nu)=\omega_{a}(\nu)^{-1 / 2} \exp \left(\int_{-\infty}^{\nu} \mathrm{d} v \frac{\chi_{0}^{\prime \prime}(v)}{\omega_{a}(v)}\right)$.

We observe that the Jacobian of the change of variables $s \rightarrow \nu=\nu_{r}$ in the integral for the probability density $P_{\tau}(u)=\int_{-\infty}^{\infty} \frac{\mathrm{d} s}{2 \pi} \mathrm{e}^{-\mathrm{i} s u}\left\langle\mathrm{e}^{\mathrm{i} s \xi_{\tau}}\right\rangle$ cancels the factor $\chi_{r}^{\prime \prime}\left(\nu_{r}\right)$ in the denominator of the expressions (7)-(9) for $\left\langle\mathrm{e}^{\mathrm{i} s \xi_{\tau}}\right\rangle$.

This allows to perform explicitly the summation over the eigenstates $r=(\mathbb{P}, \mathbb{H})$ after introducing a contour integral to enforce the constraint that the number of positive elements of $\mathbb{P}$ minus the number of negative elements of $\mathbb{P}$ is equal to the number of negative elements of $\mathbb{H}$ minus the number of positive elements of $\mathbb{H}$. In the stationary and the step case, an extra step is needed to perform the summation over $\mathbb{H}$ and reduce the determinant squared to a single determinant, using a version of the Cauchy-Binet formula,

$$
\begin{array}{r}
\sum_{\mathbb{H} \subset \Gamma} \mathbb{1}_{\{|\mathbb{H}|=|\mathbb{P}|\}} \operatorname{det}\left(L_{a, b}\right)_{\substack{a \in \mathbb{P} \\
b \in \mathbb{H}}} \operatorname{det}\left(M_{b, c}\right)_{\substack{b \in \mathbb{H} \\
c \in \mathbb{P}}} \\
=\operatorname{det}\left(\sum_{b \in \Gamma} L_{a, b} M_{b, c}\right)_{\substack{a \in \mathbb{P} \\
c \in \mathbb{P}}}
\end{array}
$$

The arbitrary set $\Gamma$ is equal to $\mathbb{Z}+1 / 2$ here. A Fredholm determinant is obtained in the end using the identity

$$
\sum_{\mathbb{P} \subset \Gamma} \operatorname{det}\left(J_{a, b}\right)_{\substack{a \in \mathbb{P} \\ b \in \mathbb{P}}}=\operatorname{det}(\mathbb{1}+J)
$$

where $J$ is an operator acting on $\Gamma$, with discrete kernel $J_{a, b}$. One recovers (10).

The expansion of the Fredholm determinant of (10)

$$
\operatorname{det}(\mathbb{1}-J)=\sum_{n=0}^{\infty} \frac{(-1)^{n}}{n !} \sum_{a_{1}, \ldots, a_{n} \in \mathbb{Z}+\frac{1}{2}} \operatorname{det}\left(J_{a_{j}, a_{k}}\right)_{j, k}
$$

leads to a corresponding expansion of the cumulative distribution function of the current, $F_{\tau}(u)=\sum_{n=0}^{\infty} \mu_{n}(\tau, u)$. Numerics in the flat case indicate that the coefficients $\mu_{n}\left(\tau, \tau^{1 / 3} u\right)$ do not converge individually when $\tau \rightarrow 0$ : only the full sum $F_{\tau}\left(\tau^{1 / 3} u\right)$ has a finite limit, the GOE Tracy-Widom distribution $F_{1}$. This is in contrast with a common situation for long time limits of finite time Fredholm determinant expressions for TASEP on $\mathbb{Z}$, where all 
the terms of the expansion converge individually and it is sufficient to take the limit of the kernel.

* sylvain.prolhac@irsamc.ups-tlse.fr

[S1] L.-H. Gwa and H. Spohn. Six-vertex model, roughened surfaces, and an asymmetric spin Hamiltonian. Phys. Rev. Lett., 68:725-728, 1992.

[S2] S. Prolhac. Spectrum of the totally asymmetric simple exclusion process on a periodic lattice - first excited states. J. Phys. A: Math. Theor., 47:375001, 2014.
[S3] K. Motegi, K. Sakai, and J. Sato. Long time asymptotics of the totally asymmetric simple exclusion process. J. Phys. A: Math. Theor., 45:465004, 2012.

[S4] S. Prolhac. Asymptotics for the norm of Bethe eigenstates in the periodic totally asymmetric exclusion process. J. Stat. Phys., 160:926-964, 2015.

[S5] S. Prolhac. Current fluctuations and large deviations for periodic TASEP on the relaxation scale. J. Stat. Mech., 2015:P11028, 2015.

[S6] N.M. Bogoliubov. Determinantal representation of the time-dependent stationary correlation function for the totally asymmetric simple exclusion model. SIGMA, 5:052, 2009. 